\title{
Lymph node retrieval after dissolution of surrounding adipose tissue for pathological examination of colorectal cancer
}

\author{
HIROMICHI MAEDA ${ }^{1}$, KEN OKAMOTO $^{1}$, KOJI OBA ${ }^{2}$, MAI SHIGA $^{3}$, YUKI FUJIEDA $^{3}$, TSUTOMU NAMIKAWA ${ }^{3}$, \\ MAKOTO HIROI $^{4}$, ICHIRO MURAKAMI ${ }^{4}$, KAZUHIRO HANAZAKI $^{3}$ and MICHIYA KOBAYASHI ${ }^{1}$ \\ ${ }^{1}$ Cancer Treatment Center, Kochi Medical School Hospital, Kochi 783-8505; ${ }^{2}$ Department of Biostatistics, \\ Graduated School of Medicine, The University of Tokyo, Tokyo 113-0033; ${ }^{3}$ Depatment of Surgery, Kochi Medical School; \\ ${ }^{4}$ Department of Diagnostic Pathology, Kochi Medical School, Kochi University, Kochi 783-8505, Japan
}

Received September 21, 2017; Accepted November 24, 2017

DOI: $10.3892 / \mathrm{ol} .2017 .7629$

\begin{abstract}
Examination of $>12$ lymph nodes (LNs) is important for the diagnostic accuracy of nodal status following resection of colorectal cancer. In the present study, the efficacy of a fat dissolution technique for $\mathrm{LN}$ retrieval was evaluated using resected colon and rectum mesentery. First, the resected mesentery was searched for LNs by inspection and palpation immediately after surgery. Subsequently, fat dissolution liquid was applied to the remnant fat and the LN search was repeated. The primary endpoint was whether the second assessment would increase the number of evaluated LNs. Recruitment of 20 patients was planned. The study was conducted after institutional review board approval and written informed consent was obtained. Among 20 participants, 1 patient was excluded because LN dissection was not performed. The median number of LNs identified at the first and second assessments was 13 and 6 , respectively, producing a significant increase in total LNs evaluated (13 vs. 20, respectively; $\mathrm{P}<0.01$; paired t-test). One positive node was identified among the additionally identified LNs $(0.9 \%, 1 / 107)$. The second assessment increased the number of LNs assessed to $>12$ in 4 patients, and although staging was not changed, the treatment was potentially altered in 2 stage II patients. The maximum diameter of the additionally obtained LNs was significantly smaller compared with those from the first assessment (4 vs. $7.7 \mathrm{~mm}$, respectively; $\mathrm{P}<0.01$; Wilcoxon signed-rank test). After the fat dissolution technique, the tumor cells were satisfactorily stained by carcinoembryonic antigen and cytokeratin-20. In conclusion, applying fat dissolution liquid to the remnant adipose tissue of the mesentery of the colon and rectum identified additional
\end{abstract}

Correspondence to: Dr Hiromichi Maeda, Cancer Treatment Center, Kochi Medical School Hospital, Kohasu, Oko-cho, Nankoku, Kochi 783-8505, Japan

E-mail: hmaeda@kochi-u.ac.jp

Key words: body mass index, lymph nodes, colorectal, metastasis
LNs. This method should be considered when insufficient LNs are identified after conventional LN retrieval.

\section{Introduction}

After surgical resection of localized colorectal cancer, the tumor stage is determined by the extent of wall invasion and lymph node (LN) involvement (1). When regional LN metastasis is pathologically confirmed, the tumor is classified as Stage III cancer of the colon and rectum. In such cases, postoperative adjuvant chemotherapy is recommended to suppress tumor recurrence, which improves overall survival (2). When the tumor invades more than the muscular layer without LN metastasis, the tumor is classified as Stage II. Although adjuvant chemotherapy for Stage II colon cancer is not a general practice due to the lack of survival benefit, a patient with a Stage II tumor showing high risk features might benefit from adjuvant chemotherapy. According to the European Society for Medical Oncology and American Society of Clinical Oncology guidelines, in addition to the pathological tumor type and more advanced wall invasion, less than 12 pathologically evaluated LNs is also considered as a risk factor for tumor recurrence $(3,4)$.

The clear reason for the relationship between a smaller number of retrieved LNs and poorer prognosis is not fully understood, although several studies point out their connection $(5,6)$. A diminished immunological response or weakened immune system may contribute to the identification of fewer LNs and tumor progression (5). Others speculate that inadequate evaluation of LNs may result in stage migration, where stage III disease is understated as Stage II. In addition to the need for elucidating the underlying cause of this relationship, studies clearly suggest that LNs need to be adequately examined in a qualitative and quantitative manner.

The method of searching for LNs in Japan is unique from other countries, where LN retrieval is performed by pathologists or pathological assistants on formaldehyde-fixed specimens $(7,8)$. In most cases in Japan, surgeons search for the LNs immediately after surgery (before fixation). The mesentery is dissected from the large intestine, and the LNs within the adipose tissue are inspected and palpated one-by-one after cutting the peritoneum and connective tissues. A nationwide 
survey held in Japan reported that this method yielded a mean LN number of 20.3 in the teaching hospitals. However, the number of LNs identified may not be sufficient when performed in non-teaching hospitals (9). We consider that this method of LN retrieval requires a certain level of experience and motivation. In addition, obesity [high body mass index (BMI)] might hamper the identification of very small LNs within the abundant adipose tissue (Y. Fujieda, unpublished data, 2017).

In this context, a simple and assured method of identifying LNs after colorectal surgery needs to be established. Thus, the present study aimed to evaluate the efficacy of a fat dissolution liquid for aiding the search for LNs in the mesentery of the colon and rectum. A fat dissolution method was originally described by Fujino et al in 2014 using the enzymes, collagenase and trypsin (10), while the present study utilizes a commercially available fat dissolution liquid composed of collagenase and lipase. As fat dissolution liquid has been rarely used in the clinical setting, we planned to evaluate whether additional LNs (which were initially overlooked) could be identified by using the fat dissolution liquid on the remnant mesentery, after LN retrieval by the conventional inspection and palpation method.

\section{Materials and methods}

Patients. A target sample size of 20 patients was chosen for the present study. Patients were included in the study if the following inclusion criteria were met: i) a diagnosis of colorectal cancer via endoscopic and/or pathological examination, requiring surgical resection with $\mathrm{LN}$ dissection; ii) $\geq 20$ years old; iii) the ability to fully understand the present study; and iv) provided written informed consent. Besides failing to meet the inclusion criteria, the patients were excluded when investigators considered it unsuitable to include the patient into this study. The study was approved by the institutional review board of Kochi Medical School (ID: 28-59), and conducted after written informed consent was provided. The trial registration number is UMIN000023892.

First LN search using the conventional method. A regional LN search was performed immediately after the operation using a manual technique, which is common practice in our institute. First, the mesentery was dissected from the large bowel (Fig. 1). The large bowel with the primary tumor was pinned on a board to facilitate the pathological diagnosis of wall invasion ( $\mathrm{T}$ factor). For the mesentery, the serous membrane was cut with scissors and the tumor-feeding arteries were exposed to classify the LNs according to the definition issued by the Japanese Society for Cancer of the Colon and Rectum (11). The connective tissue was gently cut and the LNs within the mesentery were located by inspection and palpation. Identified LNs were fixed in $10 \%$ formaldehyde and sent to the pathologist as LNs from the first search.

Second LN search after fat dissolution. A commercially available fat dissolution liquid was provided by Sysmex (Kobe, Japan) according to the study contract. The fat dissolution liquid was prepared by adding $50 \mathrm{ml}$ of water into crystallized collagenase and lipase, and then gently dissolved immediately
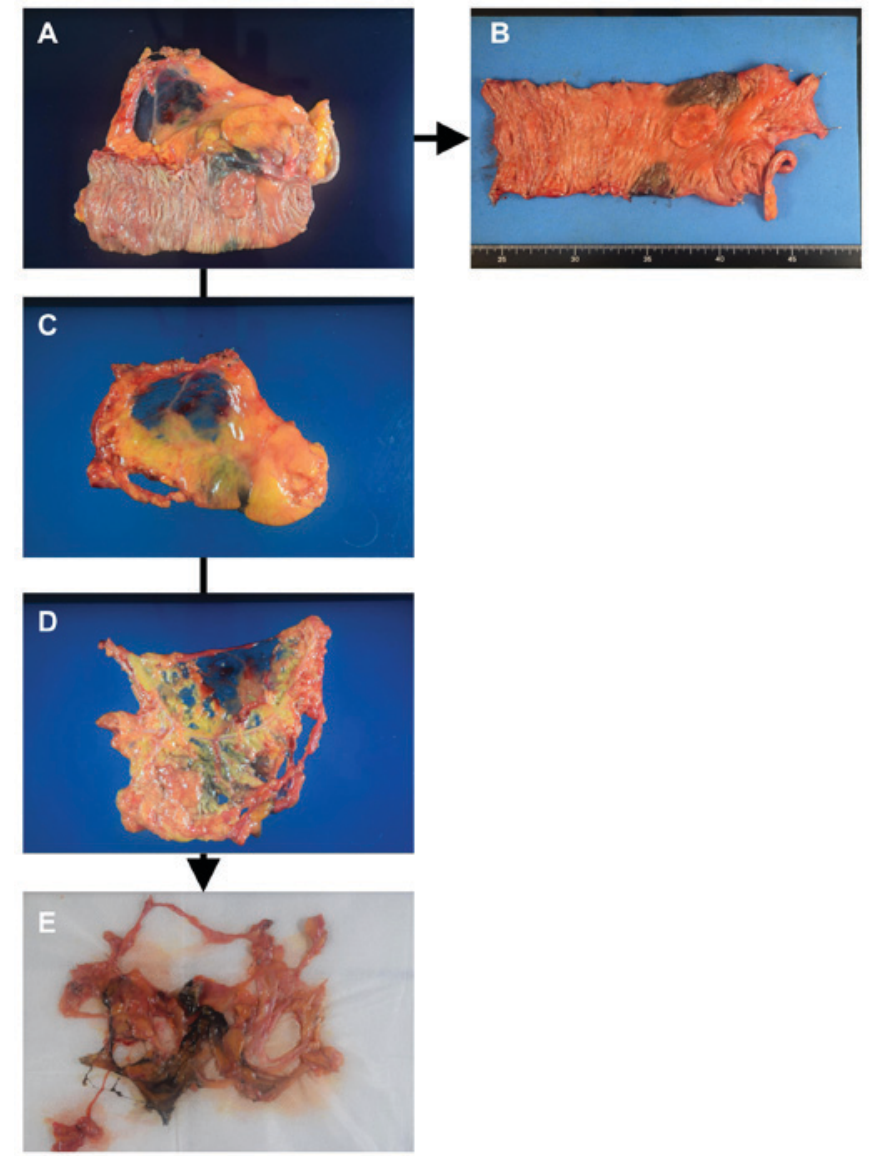

Figure 1. Conventional procedure and fat dissolution technique. (A) The resected specimen is processed immediately after surgery. (B) The large bowel with colorectal cancer is pinned on a board. (C) The mesentery is dissected from the large bowel, and the serous membrane is cut with scissors and (D) the tumor-feeding arteries are exposed and the search for regional lymph nodes (LNs) is performed. (E) After fat dissolution, the tissue is transferred onto 5-10 sheets of gauze to drain the liquid, and the tissue is searched again for LNs for pathological examination.

before use (the final concentration of collagenase and lipase was not disclosed).

The liquid was injected into the remnant adipose tissue using an $18 \mathrm{G}$ needle. The adipose tissue and liquid that leaked out of the mesentery were incubated in a water bath at $37^{\circ} \mathrm{C}$ for 40-60 $\mathrm{min}$. The tissue was then transferred onto 5-10 sheets of gauze to drain the liquid. The tissue was pressed with gauze several times in order to further push out the remaining liquid (Fig. 1E). Then, the tissue was searched again for LNs for pathological examination.

Statistical analysis. The patient sample size was calculated based on the hypothesis that the fat dissolution liquid could detect an additional $20 \%$ of LNs from the number identified at the first assessment. If the mean number of additional LNs was 3.0 (standard deviation: 4.0), the required sample size was calculated as 16 , using two-sided $\alpha=0.05$ and power $=0.8$. Finally, a target sample size of 20 patients was chosen, allowing for some ineligible cases.

In this study, the LNs identified near the colon or rectum during the microscopic examination for tumor wall invasion were excluded from the analysis. The number of LNs obtained after the first search was compared to the total number of LNs 
Table I. Patient and clinical characteristics.

\begin{tabular}{lc}
\hline Characteristics & Value \\
\hline Patient characteristics & \\
Age, mean (SD), years & $69.1(10.1)$ \\
Sex (male/female) & $7 / 12$ \\
Body mass index, mean (SD), kg/m² & $22.1(4.2)$ \\
Tumor site (right side/left side/rectum) & $9 / 6 / 4$ \\
Tumor and operative characteristics & \\
T factor (T1/T2/T3/T4) & $2 / 5 / 9 / 3$ \\
N factor (node-negative/node-positive) & $16 / 3$ \\
Distant metastasis (No/yes) & $17 / 2$ \\
Tumor size, mean (SD), cm & $44.4(21.6)$ \\
Pathological type (well/mod/muc/poor) & $6 / 10 / 1 / 2$ \\
Surgical approach (open/laparoscopic) & $2 / 17$ \\
\hline
\end{tabular}

Values are number of patients, unless otherwise indicated. SD, standard deviation; mod; moderate; muc; mucinous.

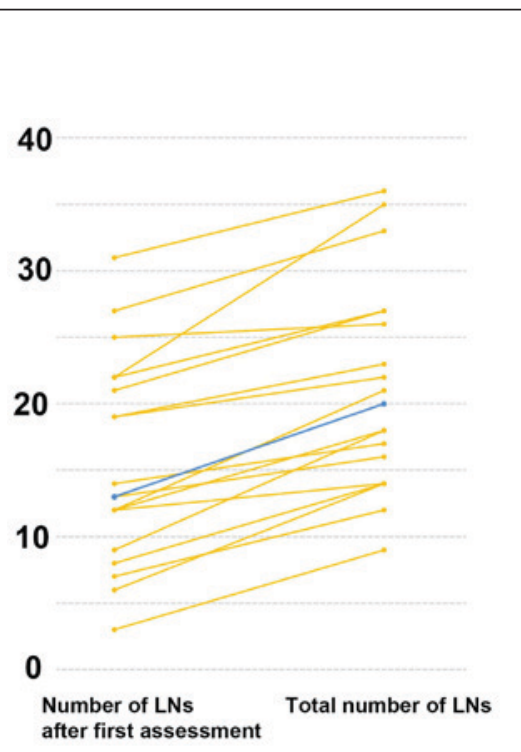

Figure 2. Comparison of the number of macroscopically identified lymph nodes (LNs) before and after the fat dissolution method. The total number of LNs found in the mesentery increased compared to the first assessment. Blue line shows median while orange lines show individual data.

(defined as the sum of the LNs identified at the first and second assessments), using a paired t test. The maximum diameters of the LNs obtained at the first and second assessments were compared using the Wilcoxon signed-rank test. A P-value of less than 0.05 was considered significant. For statistical analysis and graph depiction, Microsoft Office Excel 2007 (Microsoft Corporation,, Redmond, WA) and JMP 12.2 (SAS Institute Inc., Cary, NC, USA) were used.

\section{Results}

A total of 20 patients participated in this study between September 2016 and January 2017. The clinical features of the participating patients are presented in Table I. One patient was excluded from the analysis because LN dissection was not performed due to an oncological reason (Table I). Of the
Table II. Number of LNs obtained from first and second assessments and the number of LN metastases.

\begin{tabular}{|c|c|c|c|c|c|c|}
\hline \multirow[b]{2}{*}{ Patient } & \multirow[b]{2}{*}{ Stage } & \multicolumn{2}{|c|}{$\begin{array}{c}\text { First } \\
\text { assessment }\end{array}$} & \multicolumn{2}{|c|}{$\begin{array}{c}\text { Second } \\
\text { assessment }\end{array}$} & \multirow[b]{2}{*}{$\begin{array}{l}\text { Total } \\
\text { LNs }\end{array}$} \\
\hline & & LNs & $\begin{array}{c}\text { Positive } \\
\text { nodes }\end{array}$ & LNs & $\begin{array}{c}\text { Positive } \\
\text { nodes }\end{array}$ & \\
\hline $1^{\mathrm{a}}$ & I & 6 & 0 & 8 & 0 & 14 \\
\hline $2^{\mathrm{a}}$ & I & 8 & 0 & 6 & 0 & 14 \\
\hline 3 & I & 13 & 0 & 3 & 0 & 16 \\
\hline 4 & I & 19 & 0 & 3 & 0 & 22 \\
\hline 5 & I & 19 & 0 & 4 & 0 & 23 \\
\hline 6 & I & 22 & 0 & 5 & 0 & 27 \\
\hline 7 & I & 22 & 0 & 13 & 0 & 35 \\
\hline $8^{\mathrm{a}}$ & II & 7 & 0 & 5 & 0 & 12 \\
\hline $9^{a}$ & II & 9 & 0 & 9 & 0 & 18 \\
\hline 10 & II & 12 & 0 & 2 & 0 & 14 \\
\hline 11 & II & 12 & 0 & 6 & 0 & 18 \\
\hline 12 & II & 12 & 0 & 9 & 0 & 21 \\
\hline 13 & II & 14 & 0 & 3 & 0 & 17 \\
\hline 14 & II & 25 & 0 & 1 & 0 & 26 \\
\hline 15 & II & 27 & 0 & 6 & 0 & 33 \\
\hline 16 & II & 31 & 0 & 5 & 0 & 36 \\
\hline 17 & III & 13 & 1 & 7 & 1 & 20 \\
\hline 18 & IV & 3 & 2 & 6 & 0 & 9 \\
\hline 19 & IV & 21 & 1 & 6 & 0 & 27 \\
\hline $\begin{array}{l}\text { Median } \\
\text { (range) }\end{array}$ & - & $\begin{array}{c}13 \\
(3-31)\end{array}$ & - & $\begin{array}{c}6 \\
(1-13)\end{array}$ & - & $\begin{array}{c}20 \\
(9-36)\end{array}$ \\
\hline $\begin{array}{l}\text { Mean } \\
(\mathrm{SD})\end{array}$ & - & $\begin{array}{l}15.5 \\
(7.7)\end{array}$ & - & $\begin{array}{c}5.6 \\
(2.8)\end{array}$ & - & $\begin{array}{l}21.2 \\
(7.8)\end{array}$ \\
\hline
\end{tabular}

Patients are sorted according to the pathological tumor stage and the number of LNs obtained at the first assessment. ${ }^{\text {In }}$ these patients, the LN number after the first assessment was less than 12, but reached a sufficient level after the second assessment using fat dissolution. SD, standard deviation; LNs, lymph nodes.

remaining 19 patients, 7 were male, 6 had rectal cancer, and 17 underwent laparoscopic surgery. The mean age of the patients was 69.1 years and their mean BMI was $22.1 \mathrm{~kg} / \mathrm{m}^{2}$ (Table I).

\section{Primary endpoint}

Number of LNs identified at first and second assessments. The median number of LNs identified at the first search was 13 (range, 3-31), while the second search identified a median of 6 additional LNs (range, 1-13; Table II). Consequently, a paired t test found a significant difference between the number of LNs found at the first search and the total number of LNs found in the mesentery (13 vs. 20, respectively; $\mathrm{P}<0.01$; Fig. 2).

\section{Secondary endpoints}

Rate of positive LNs. Only one positive node was identified among the additionally identified LNs. Therefore the rate of positive nodes among the additional LNs was $0.9 \%(1 / 107)$, while that of the first assessment was $1.4 \%$ (4/295). 
Table III. Diameter of LNs obtained at the first and second assessments.

\begin{tabular}{|c|c|c|c|c|}
\hline \multirow[b]{2}{*}{ Patient } & \multicolumn{2}{|c|}{ First assessment } & \multicolumn{2}{|c|}{ Second assessment } \\
\hline & $\begin{array}{c}\text { Maximum } \\
\text { diameter }(\mathrm{mm})\end{array}$ & $\begin{array}{c}\text { Minimum } \\
\text { diameter }(\mathrm{mm})\end{array}$ & $\begin{array}{c}\text { Maximum } \\
\text { diameter }(\mathrm{mm})\end{array}$ & $\begin{array}{c}\text { Minimum } \\
\text { diameter }(\mathrm{mm})\end{array}$ \\
\hline 1 & 5.1 & 1.5 & 4.0 & 1.0 \\
\hline 2 & 6.9 & 1.2 & 4.0 & 1.1 \\
\hline 3 & 8.2 & 1.0 & 4.2 & 1.3 \\
\hline 4 & 12.6 & 2.5 & 5.0 & 2.0 \\
\hline 5 & 7.7 & 1.3 & 4.0 & 2.1 \\
\hline 6 & 7.1 & 0.9 & 6.7 & 2.2 \\
\hline 7 & 4.4 & 0.5 & 2.1 & 0.9 \\
\hline 8 & 9.0 & 1.8 & 4.2 & 2.1 \\
\hline 9 & 4.7 & 1.0 & 4.0 & 1.2 \\
\hline 10 & 4.0 & 0.9 & 2.6 & 2.3 \\
\hline 11 & 6.4 & 1.0 & 4.0 & 2.0 \\
\hline 12 & 14.3 & 4.0 & 9.0 & 3.6 \\
\hline 13 & 5.9 & 1.5 & 2.7 & 1.8 \\
\hline 14 & 11.0 & 1.0 & 2.4 & - \\
\hline 15 & 7.3 & 1.1 & 4.0 & 0.9 \\
\hline 16 & 11.7 & 0.9 & 7.2 & 1.8 \\
\hline 17 & 11.7 & 1.0 & 4.9 & 2.0 \\
\hline 18 & 14.0 & 4.1 & 2.8 & 1.5 \\
\hline 19 & 8.5 & 1.5 & 5.0 & 2.1 \\
\hline Median (range) & $7.7(4-14.3)$ & $1.1(0.5-4.1)$ & $4.0(2.1-9)$ & $1.9(0.9-3.6)$ \\
\hline Mean (SD) & $8.4(3.2)$ & $1.5(1.0)$ & $4.4(1.7)$ & $1.8(0.7)$ \\
\hline
\end{tabular}

Patients are sorted according to the pathological tumor stage and the number of LNs obtained at the first assessment (same as Table II). SD, standard deviation; LNs, lymph nodes.
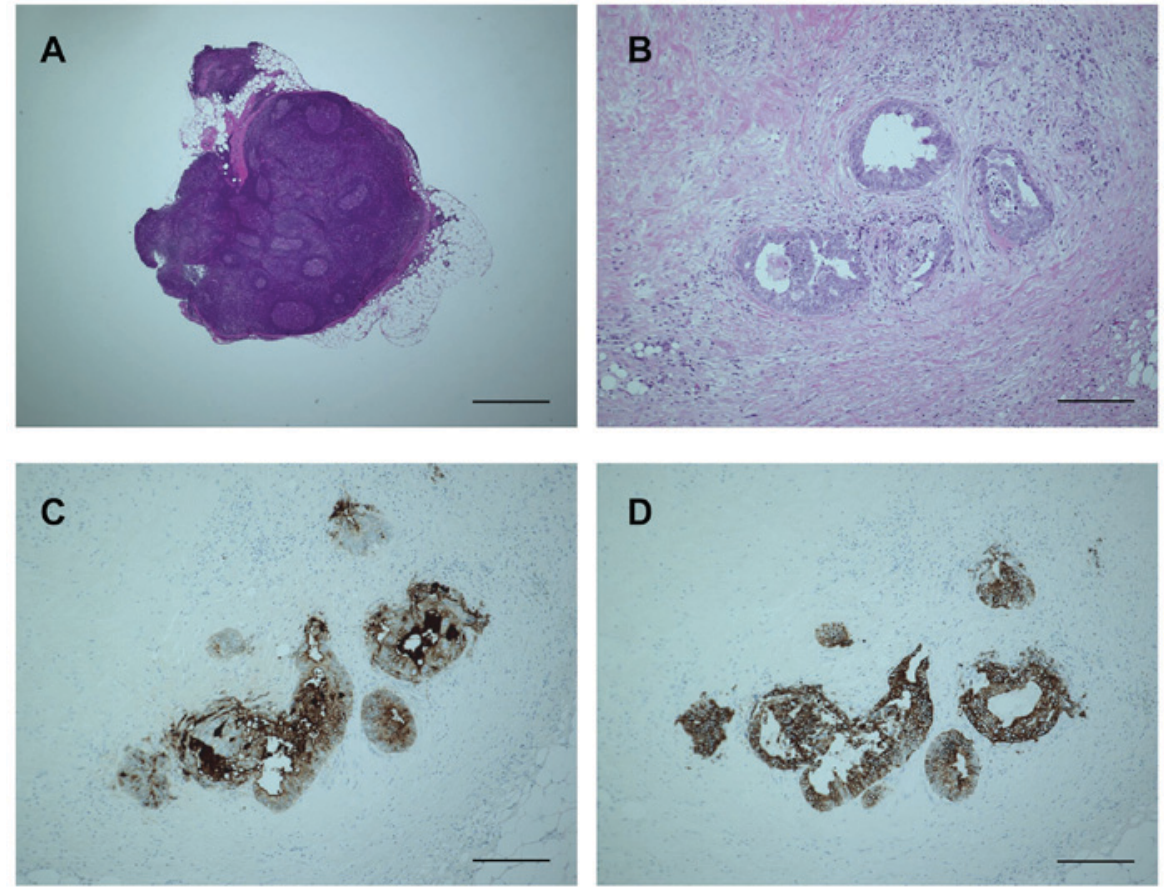

Figure 3. Microscopic examination of obtained lymph node (LN) and cancer cells after fat dissolution. (A) The structure of the LN is well-maintained even after $60 \mathrm{~min}$ incubation in the fat dissolution liquid. The bar is $1,000 \mu \mathrm{m}$. (B) Hematoxylin and eosin staining of the tumor nodule is also not hampered by fat dissolution. The bar is $200 \mu \mathrm{m}$. (C and D) The tumor nodule is clearly positive for (C) carcinoembryonic antigen and (D) cytokeratin-20, compatible with the control results obtained at the first LN assessment (data not shown). The bars are $200 \mu \mathrm{m}$. 
Influence on treatment. Tumor staging was not altered by the additional search for LNs. However, in four patients without distant metastasis, the second search using the fat dissolution technique had an impact on the adequacy of identified LNs, in terms of the minimum number required for pathological examination (minimum of 12). In two patients with Stage I cancer and two with Stage II cancer, less than 12 LNs were identified at the first search (patient numbers 1, 2, 8 and 9 in Table II). In the two patients with Stage I cancer, their subsequent treatment would not have been influenced by the lack of an additional LN search. However, in the two patients with Stage II colorectal cancer, the tumor could have been diagnosed as high-risk Stage II, and it would have been possible that adjuvant chemotherapy was started in these two patients if the second LN search had not been performed.

Maximum diameter of the additional LNs. The median maximum diameter of the LNs obtained after the second search was significantly smaller than the maximum diameter of the LNs obtained at the first search (4 vs. $7.7 \mathrm{~mm}$, respectively; $\mathrm{P}<0.01$, Wilcoxon signed-rank test; Table III). Meanwhile, the minimum diameter of the LNs identified in the first and second assessments did not differ significantly (1.9 vs. $1.1 \mathrm{~mm}$, respectively; $\mathrm{P}=0.35$; Table $\mathrm{III}$ ).

Staining for tumor-specific antigens. Because only one patient had a tumor nodule among the additionally obtained LNs, only one nodule was evaluated. As there was no clear histological evidence of a residual $\mathrm{LN}$ in the nodule, the node was diagnosed as a tumor nodule according to the definition of the Japanese Classification of Cancer in the Colon and Rectum $(1,12)$, and the Union for International Cancer Control-TNM classification. Immunohistochemistry clearly revealed that the tumor cells were positive for carcinoembryonic antigen, cytokeratin-20 and pankeratin (Fig. 3). These results suggest that the fat dissolution method does not alter the stainability of tumor-specific antigens.

\section{Discussion}

This study clearly demonstrates that the fat dissolution technique increases the total number of examined LNs when applied to the remnant adipose tissue of the mesentery. In the present study, the median and mean number of LNs obtained at the first assessment was 13 and 15.6, respectively. A statistical comparison between our previous data (12) and the results of this study was not performed because the background characteristics significantly influenced the number of LNs retrieved (5). However, we note that the median LN number of 13 was quite similar to our previous data. Moreover, 13 of 17 patients $(76.5 \%)$ with localized colorectal cancer received adequate LN examination, which surpasses the rate reported from non-teaching hospitals in the US, and is similar to that reported from specialized institutes (13). We consider that these two points suggest that the quality of the first LN search was adequate. Nevertheless, the fat dissolution technique applied to the remnant mesentery yielded additional LNs, and as a result of the whole procedure, LN examination was sufficiently performed in all but one patient with Stage IV disease.
The comparison of the maximum diameters of LNs obtained from the first and second assessments showed that the residual LNs were smaller than those obtained with the conventional method. However, searching for smaller LNs can still have a significant clinical impact. Rodriguez-Bigas et al retrospectively studied the size of LNs in patients with Stage III colorectal cancer, identifying 253 positive nodes out of 3087 LNs. Among the positive nodes, they reported that $69 \%$ were $5 \mathrm{~mm}$ or less in diameter (14). Similarly, Rollvén et al reported that $66 \%$ of positive nodes were less than $4 \mathrm{~mm}$ (15). Although tumor staging was not influenced by the fat dissolution technique in the present study, these reports highlight the importance of the examination of an adequate number of LNs, irrespective of the size of the LNs.

The most significant drawback of the fat dissolution technique introduced in the present study is the incubation time. The incubation time could be reduced by modifying the concentrations of collagenase and lipase (10), which is hampered by the increased cost so far. The conventional method itself requires approximately 30-60 min to be performed (10), followed by 40-60 min of incubation time for fat dissolution, and then the second assessment. Therefore, this time-consuming procedure would be quite difficult to perform in all cases as a routine practice. Alternatively, it could be applied only to cases where a low number of LNs have been retrieved by the conventional method, or in patients where $\mathrm{LN}$ retrieval is expected to be difficult due to high a BMI (Y. Fujieda, unpublished data, 2017). Another disadvantage is that this method can only be used in specimens before formaldehyde fixation.

Besides the small number of recruited patients, one limitation of the present study was that the fat dissolution technique was applied only to the remnant mesentery after the initial conventional assessment. Although this technique could be directly applied to the mesentery without a conventional LN search, the efficacy of the technique under this setting has yet to be determined. Another limitation is that only one metastatic node was obtained after using the fat dissolution technique. While hematoxylin and eosin staining and immunohistochemistry staining demonstrated that fat dissolution does not hamper microscopic examination, further study would be necessary to confirm this finding. To address these points, another clinical study is currently underway (UMIN ID: UMIN000029448).

In conclusion, the present study demonstrates that our fat dissolution technique yields additional LNs for examination when used on the remnant mesentery of the colon and rectum. Specifically, this method should be considered when the number of identified LNs is insufficient after conventional LN retrieval.

\section{Acknowledgements}

The authors would like to thank Ms. Nishimori and Ms. Yamaguchi for their assistance with data management.

\section{References}

1. Union for International Cancer Control: TNM Classification of Malignant Tumours. Sobin LH, Gospodarowicz MK and Wittekind C (eds). 7th edition. Wiley-Blackwell, Hoboken, NJ, 2010 . 
2. Sargent DJ, Patiyil S, Yothers G, Haller DG, Gray R, Benedetti J, Buyse M, Labianca R, Seitz JF, O'Callaghan CJ, et al: End points for colon cancer adjuvant trials: Observations and recommendations based on individual patient data from 20,898 patients enrolled onto 18 randomized trials from the ACCENT Group. J Clin Oncol 25: 4569-4574, 2007.

3. Benson AB III, Schrag D, Somerfield MR, Cohen AM, Figueredo AT, Flynn PJ, Krzyzanowska MK, Maroun J, McAllister P, Van Cutsem E, et al: American society of clinical oncology recommendations on adjuvant chemotherapy for stage II colon cancer. J Clin Oncol 22: 3408-3419, 2004.

4. Schmoll HJ, Van Cutsem E, Stein A, Valentini V, Glimelius B, Haustermans K, Nordlinger B, van de Velde CJ, Balmana J, Regula J, et al: ESMO consensus guidelines for management of patients with colon and rectal cancer. A personalized approach to clinical decision making. Ann Oncol 23: 2479-2516, 2012.

5. Märk1 B: Stage migration vs. immunology: The lymph node count story in colon cancer. World J Gastroenterol 21: 12218-12233, 2015.

6. Parsons HM, Tuttle TM, Kuntz KM, Begun JW, McGovern PM and Virnig BA: Association between lymph node evaluation for colon cancer and node positivity over the past 20 years. JAMA 306: 1089-1097, 2011.

7. Kuo YH, Lee KF, Chin CC, Huang WS, Yeh $\mathrm{CH}$ and Wang JY: Does body mass index impact the number of LNs harvested and influence long-term survival rate in patients with stage III colon cancer? Int J Colorectal Dis 27: $1625-1635,2012$.
8. Linebarger JH, Mathiason MA, Kallies KJ and Shapiro SB: Does obesity impact lymph node retrieval in colon cancer surgery? Am J Surg 200: 478-482, 2010.

9. Ueno H, Hase K, Hashiguchi Y, Shinto E, Shimazaki H, Yamamoto J, Nakamura T and Sugihara K: Potential causes of stage migration and their prognostic implications in colon cancer: A nationwide survey of specialist institutions in Japan. Jpn J Clin Oncol 44: 547-555, 2014.

10. Fujino S, Miyoshi N, Ohue M, Noura S, Tomita Y, Yano M and Sakon M: New enhanced and effective method for staging cancer to detect lymph nodes after fat-dissociation. Oncol Rep 32: 922-926, 2014.

11. Japanese Society for Cancer of the Colon and Rectum: Japanese Classification of Colorectal Carcinoma. 8th edition. Kanehira-Syuppan, Tokyo, 2013 (In Japanese).

12. Shiga M, Maeda H, Oba K, Okamoto K, Namikawa T, Fujisawa K, Yokota K, Kobayashi M and Hanazaki K: Safety of laparoscopic surgery for colorectal cancer in patients over 80 years old: A propensity score matching study. Surg Today 47: 951-958, 2017.

13. Baxter NN, Virnig DJ, Rothenberger DA, Morris AM, Jessurun J and Virnig BA: Lymph node evaluation in colorectal cancer patients: A population-based study. J Natl Cancer Inst 97: 219-225, 2005.

14. Rodriguez-Bigas MA, Maamoun S, Weber TK, Penetrante RB, Blumenson LE and Petrelli NJ: Clinical significance of colorectal cancer: Metastases in lymph nodes $<5 \mathrm{~mm}$ in size. Ann Surg Oncol 3: 124-130, 1996.

15. Rollvén E, Abraham-Nordling M, Holm T and Blomqvist L: Assessment and diagnostic accuracy of lymph node status to predict stage III colon cancer using computed tomography. Cancer Imaging 17: 3, 2017. 